\title{
Epigenetic changes in renal genes dysregulated in mouse and rat models of type 1 diabetes
}

\author{
Radko Komers ${ }^{1}$, Daniel Mar², Oleg Denisenko², Bei Xu ${ }^{1}$, Terry T Oyama and Karol Bomsztyk²
}

Epigenetic processes are increasingly being recognized as factors in the pathophysiology of diabetes complications, but few chromatin studies have been done in diabetic nephropathy (DN). We hypothesized that changes in mRNA expression of DN-related genes are associated with epigenetic alterations and aberrant expression of histone-modifying enzymes. RT-PCR and a matrix-chromatin immunoprecipitation platform were used to examine renal mRNA expression, RNA polymerase II (Pol II) recruitment, and epigenetic marks at DN-related genes in the mouse (OVE26) and streptozotocininduced rat models of type 1 diabetes. Diabetes induced renal expression of Cox2, S100A4/FSP-1, and vimentin genes in both the mouse and the rat models of DN. Mcp-1 and laminin $\gamma 1$ (Lamc1) expression were increased in diabetic mice but not in rats. Comparison of mRNA and Pol II levels suggested that the diabetes-induced expression of these transcripts is mediated by transcriptional and posttranscriptional processes. Decreases in histone H3 lysine 27 tri-methylation ( $\mathrm{H} 3 \mathrm{~K} 27 \mathrm{~m} 3$, silencing mark) and increases in $\mathrm{H} 3$ lysine 4 di-methylation (H3K4m2, activating mark) levels were the most consistent epigenetic alterations in the tested genes. In agreement with these results, immunoblot analysis showed increased protein abundance of renal $\mathrm{H} 3 \mathrm{~K} 27 \mathrm{~m} 2 / 3$ demethylase $\mathrm{KDM} 6 \mathrm{~A}$, but no changes in cognate methyltransferase Ezh2 in kidneys of the OVE26 mice compared with controls. In diabetic rats, Ezh2 expression was higher without changes in KDM6A, demonstrating that mechanisms of DN-induced H3K27m3 loss could be species specific. In summary, we show that altered mRNA expression of some DN-related genes is associated with changes in Pol II recruitment and a corresponding decrease in repressive $\mathrm{H} 3 \mathrm{~K} 27 \mathrm{~m} 3$ at the selected loci, and at least in mice with equivalent changes in renal expression of cognate histone-modifying enzymes. This pattern could contribute to diabetes-mediated transitions in chromatin that facilitate transcriptional changes in the diabetic kidney.

Laboratory Investigation (2013) 93, 543-552; doi:10.1038/labinvest.2013.47; published online 18 March 2013

KEYWORDS: diabetic nephropathy; histone enzymes; histone modifications; RNA polymerase II

Chromatin modifications may have an important role during the initiation and progression of diabetic nephropathy $(\mathrm{DN})^{1-11}$ by altering the expression of genes involved in this disease. Pioneering work by Natarajan and coworkers ${ }^{2,11}$ has suggested alterations in epigenetic control of inflammatory gene responses in the cardiovascular system (CVS). They also found an epigenetic basis for atherogenic and proinflammatory phenotypes of vascular smooth muscle cells derived from type 2 diabetic $\mathrm{db} / \mathrm{db}$ mice. In parallel, El-Osta et $a l .{ }^{5}$ have shown that transient hyperglycemia resulted in increased RelA/p65 NF-кB subunit expression, long-lasting activating epigenetic histone marks, and cognate enzyme alterations at the RelA/p65 promoter in aortic endothelial cells both in vitro and in nondiabetic mice. ${ }^{1,5}$
$\mathrm{DN}$ is the most serious microvascular complication of type 1 diabetes (T1D). Yet, compared with cardiovascular disease, diabetes-induced chromatin changes in the kidney have been less studied. Sun et al. ${ }^{12}$ examined rat mesangial cells treated with transforming growth factor- $\beta$ and high glucose in vitro. They found increased expression of the methyltransferase SET7/9, its recruitment to prosclerotic genes, and changes in histone $\mathrm{H} 3$ lysines methylation at these genes. These results suggested diabetes-induced epigenetic alterations that might contribute to elevated expression of prosclerotic genes in mesangial cells.

These studies represent important advances in the diabetes field, but analysis of chromatin marks along DN-relevant genes in vivo has not been done. We hypothesized that

\footnotetext{
${ }^{1}$ Division of Nephrology and Hypertension, Oregon Health and Science University, Portland, OR, USA and ${ }^{2}$ UW Medicine Lake Union, University of Washington, Seattle, WA, USA

Correspondence: Professor K Bomsztyk, MD, UW Medicine Lake Union, University of Washington, 850 Republican Street, Box 358050, Seattle, WA 98109, USA. E-mail: karolb@u.washington.edu 
changes in gene expression in the diabetic kidney are associated with altered chromatin modifications. The OVE26 mice $^{13}$ and streptozotocin (STZ)-induced rats are two different models of insulinopenic diabetes that develop a variety of renal changes that share some features with human disease, including common sets of aberrantly expressed genes. We used these two experimental models to investigate whether altered renal gene expression in the diabetic milieu is associated with corresponding epigenetic changes.

We examined genes involved in diabetes-induced low-grade chronic inflammation and prosclerotic/profibrotic processes. Our gene selection was based on previous evidence by others, suggesting their epigenetic control in non-renal tissues in diabetes, ${ }^{1,5}$ on our recent studies indicating their strong epigenetic control in nondiabetic kidney disorders, ${ }^{14-15}$ as well as on our recently published profile of genes upregulated during the diabetes-induced renal tubulointerstitial fibrosis. ${ }^{16}$

Specifically, we measured mRNA expression, polymerase II (Pol II) density, and histone marks of open and closed chromatin at $\operatorname{Cox} 2,{ }^{17-19}$ MCP-1, ${ }^{20}$ S100A4/fibroblaststimulating factor (s100A4/FSP-1), ${ }^{21}$ vimentin (Vim), ${ }^{22}$ and laminin $\gamma 1^{23}$ genes known to be upregulated in the diabetic kidney. We also analyzed the expression of histone-modifying enzymes that could catalyze observed chromatin changes.

\section{MATERIALS AND METHODS Reagents}

Bovine serum albumin, phosphate-buffered saline, salmon sperm DNA, and protein A were from Sigma, and proteinase $\mathrm{K}$ was from Invitrogen. Matrix ChIP 96-well polypropylene plates were from Bioexpress. Formaldehyde, ethanol, $\mathrm{NaCl}$, EDTA, Triton X-100, NP-40, Tris-HCl, leupeptin, PMSF, p-nitrophenyl phosphate, $\mathrm{NaF}, \mathrm{Na}_{3} \mathrm{VO}_{4}, \mathrm{Na}_{2} \mathrm{MoO}_{4}$, and $\beta$ glycerophosphate were from Sigma.

\section{Animal Models of Diabetes OVE26 mice}

Studies were conducted in previously characterized OVE26 mice, a model of T1D. ${ }^{13,24}$ OVE26 animals express a chicken calmodulin minigene controlled by the rat insulin II promoter and develop hyperglycemia within $24 \mathrm{~h}$ of age because of decreased pancreatic insulin. ${ }^{13}$ OVE26 animals exhibit severe albuminuria and, at later stages, renal structural changes resembling human DN. ${ }^{13,25}$ Male OVE26 mice on the FVB background and control FVB mice were obtained from the Jackson Laboratories ( $n=6$ in each group) were studied at 8 weeks of age.

Mice were anesthetized with intraperitoneal (i.p.) injection of Inactin $(100 \mathrm{mg} / \mathrm{kg}$ body weight), blood samples for determinations of blood glucose (BG) were obtained from the abdominal aorta, and the kidneys were removed, decapsulated, the cortical portions separated, snap frozen in liquid nitrogen, and stored at $-80^{\circ} \mathrm{C}$ for further analyses by Matrix ChIP, RT-PCR, and protein expression studies.

\section{STZ-treated diabetic rat}

Parallel studies were performed in adult male Sprague-Dawley rats with initial weights $\sim 300 \mathrm{~g}$. The rats were made diabetic by i.p. injection of STZ (Sigma, St Louis, MO, USA), $65 \mathrm{mg} / \mathrm{kg}$ body weight. Diabetic rats received to 2-4 IU of long-acting insulin/day (Lantus; Eli Lilly, Indianapolis, IN) to maintain BG at $\sim 400 \mathrm{mg} / \mathrm{dl}$ and secure long-term survival. Age-matched nondiabetic Sprague-Dawley rats served as controls. All rats were fed standard rat chow (Rodent Laboratory Chow 5001; Ralston Purina, Richmond, IN) ad libitum. The animals were studied at 4 weeks after induction of diabetes. At this time point, the rats were anesthetized with i.p. injection of Inactin $(100 \mathrm{mg} / \mathrm{kg}$ body weight), and blood samples for determinations of BG and glycosylated hemoglobin (HbAlc) were obtained from the abdominal aorta, followed by renal cortex collections and processing as described above.

All studies involving animals were approved by the Portland Veteran Affairs Institutional Animal Care and Use Subcommittee.

\section{RNA Extraction and CDNA Synthesis}

RNA was extracted from tissue fragments using Trizol reagent as per the manufacturer's protocol. To synthesize cDNA, $400 \mathrm{ng}$ of Trizol-extracted total RNA was used in reverse transcription reactions with $200 \mathrm{U}$ MMLV reverse transcriptase (Invitrogen) and random hexamers. RT reactions were diluted 100 -fold before running $\mathrm{qPCR}{ }^{26}$

\section{Chromatin Preparation and Multiplex Matrix ChIP}

For ChIP assays, tissue fragments were cross-linked with formaldehyde, and chromatin was sheared using Diagenode Bioruptor. ChIP assays were performed using the multiplex Matrix ChIP platform in protein A-coated 96-well polypropylene microplates as described before ${ }^{14,27,28}$ using pulldown antibodies raised against Pol II CTD (4H8) (Santa Cruz Biotechnology; catalog no. Sc-47701), H3 lysine 4 dimethylation (H3K4m2; Millipore; catalog no. 07-030), H3K4m3 (Millipore; catalog no. 04-745), and H3 lysine 27 tri-methylation (H3K27m3; Abcam, Cambridge, MA, USA; catalog no. Ab6002). A volume of $1-2 \mu \mathrm{l}$ of eluted DNA was used in $2-4 \mu \mathrm{l}$ real-time PCR reactions (ABI7900HT). All PCR reactions were run in triplicates. PCR primers were designed using the Primer3 software (http://frodo.wi.mit. edu/) and shown in Table 1.

\section{Immunoblotting}

Tissue preparation and western blot analysis were performed as previously described ${ }^{17,29}$ using the primary antibody raised against KDM6A (Abcam) or Ezh2 (Upstate, Billerica, MA, USA).

\section{Statistical Analysis}

Comparisons of variables between the control and diabetic groups were analyzed by $t$-test. Statistical significance was defined as $P<0.05$. 
Table 1 PCR primers

\begin{tabular}{|c|c|c|c|}
\hline Species & Gene & Forward & Reverse \\
\hline Mouse & $\operatorname{cox} 2$ & $5^{\prime}$-TTAAGGCATCACAGTCCGAG-3' & 5'-TTGAATGTGAAGTTGACCCG-3' \\
\hline Mouse & S100A4/FSP-1 & 5'-GGTGTAGTTGCCAGTGGGGG-3' & $5^{\prime}$-TGCGAAGAAGCCAGAGTAAGGC-3' \\
\hline Mouse & MCP-1 & $5^{\prime}$-GAATGAAGGTGGCTGCTATG-3' & $5^{\prime}$-AACCCAGAAACATCCAATTCTC-3' \\
\hline Mouse & Lamc1 & $5^{\prime}$-GGAACCTTCCCACACGGGTC-3' & $5^{\prime}$-CGGTGCTGATGCCCTCAAGT-3' \\
\hline Mouse & $\beta$-actin & 5'-GTCCACCTTCCAGCAGATGT-3' & 5'-AAGCCATGCCAATGTTGTCT-3' \\
\hline Rat & $\operatorname{cox} 2$ & 5'-TGCAGAGTTGAAAGCCCTCT-3' & $5^{\prime}$-CCCCAAAGATAGCATCTGGA-3' \\
\hline Rat & CypA & 5'-GTGGTCTITGGGAAGGTGAA-3' & $5^{\prime}$-TACAGGGTATTGCGAGCAGA-3' \\
\hline Rat & S100A4/FSP-1 & 5'-AGGACAGACGAAGCTGCATT-3' & $5^{\prime}-$ CTCACAGCCAACATGGAAGA-3' \\
\hline Rat & $\beta$-actin & $5^{\prime}$-CTCTGAACCCTAAGGCCAAC-3' & $5^{\prime}-$ GACCAGAGGCATACAGGGAC-3' \\
\hline
\end{tabular}

Table 2 Physical and metabolic parameters of control (FVB) and diabetic OVE26 mice

\begin{tabular}{lllllc} 
Group & BW (g) & LKW $(\mathrm{mg})$ & BG $(\mathrm{mg} / \mathrm{dl})$ & HbA1c (\%) & ACR $(\mathrm{mg} / \mathrm{mM})$ \\
\hline FVB & $27.5 \pm 0.4$ & $225 \pm 3$ & $166 \pm 22$ & $3.0 \pm 0.2$ & $26.5 \pm 9.5$ \\
OVE26 & $20.1 \pm 0.4^{\dagger}$ & $272 \pm 13^{*}$ & $605 \pm 25^{\dagger}$ & $5.9 \pm 0.2^{\dagger}$ & $174.5 \pm 14.7^{\dagger}$
\end{tabular}

Abbreviations: ACR, albumin/creatinine ratio; BG, blood glucose; BW, body weight; HbA1c, glycosylated hemoglobin; LKW, left kidney weight.

${ }^{*} P<0.05,{ }^{\dagger} P<0.01$ vs FVB.

\section{RESULTS}

\section{Physical and Metabolic Parameters}

OVE26 mice displayed kidney hypertrophy and severe hyperglycemia (Table 2). Similar to diabetic mice, diabetic rats demonstrated slower weight gain, renal hypertrophy, and increased BG and HbAlc (Table 3).

\section{Changes in Renal mRNA Expression, Pol II Recruitment, and Histone Marks}

Expression of several pro-inflammatory genes, such as COX2 and $M C P-1$, has previously been found to be increased in DN models. ${ }^{18,20,30,31}$ In addition, chromatin mechanisms have been implicated in the induction of these genes in CVS of experimental diabetes. ${ }^{2}$

\section{Cyclooxygenase 2}

Mouse cyclooxygenase 2 (mCox 2$)$ mRNA expression was increased in diabetic OVE26 as compared with age-matched FVB control animals $(P<0.05$, Figure 1 , row 1$)$. The differences in rat $\operatorname{Cox} 2(\mathrm{rCox} 2)$ expression were also observed
Table 3 Physical and metabolic parameters of control and diabetic rats

\begin{tabular}{lcccc}
\hline Group & BW (g) & LKW (g) & BG (mg/dl) & HbA1c (\%) \\
\hline Control & $351 \pm 9$ & $1.35 \pm 0.06$ & $97 \pm 2$ & $3.43 \pm 0.05$ \\
DM & $267 \pm 16^{\dagger}$ & $1.41 \pm 0.08$ & $499 \pm 14^{\dagger}$ & $7.20 \pm 0.08^{\dagger}$
\end{tabular}

Abbreviations: BG, blood glucose; BW, body weight; DM, diabetic; HbA1C, glycosylated hemoglobin; LKW, left kidney weight. ${ }^{\dagger} P<0.01$.

between diabetic rats and their respective controls $(P<0.05$, Figure 1, row 1).

Gene transcription can be regulated at either initiation, elongation, or both steps. ${ }^{32}$ Observation of high peaks of Pol II density at the $5^{\prime}$ ends and relatively low levels along genes supports the notion that transition of the promoter-bound Pol II to elongation is one of the key limiting steps in transcription. $^{28,33}$ Therefore, we measured Pol II levels engaged in elongation at the $3^{\prime}$ ends of selected genes, which are a better estimate of rates of mRNA synthesis. The canonical permissive marks $\mathrm{H} 3 \mathrm{~K} 4 \mathrm{~m} 2$ and $\mathrm{H} 3 \mathrm{~K} 4 \mathrm{~m} 3$ are enriched at $5^{\prime}$ ends of genes but are also found all along transcribed regions at levels that positively correlate with gene expression. ${ }^{33}$ The canonical repressive mark, H3K27m3, is found at approximately the same level along genes and is inversely correlated with gene expression. ${ }^{33}$ In mice and rats, there were no statistically significant differences in Pol II levels at Cox 2 gene between the diabetic and control animals. In mice, increase in mRNA expression was matched by loss of H3K27m3 $(P<0.01$, Figure 1, row 5). There were no changes in histone marks in diabetic rats. 


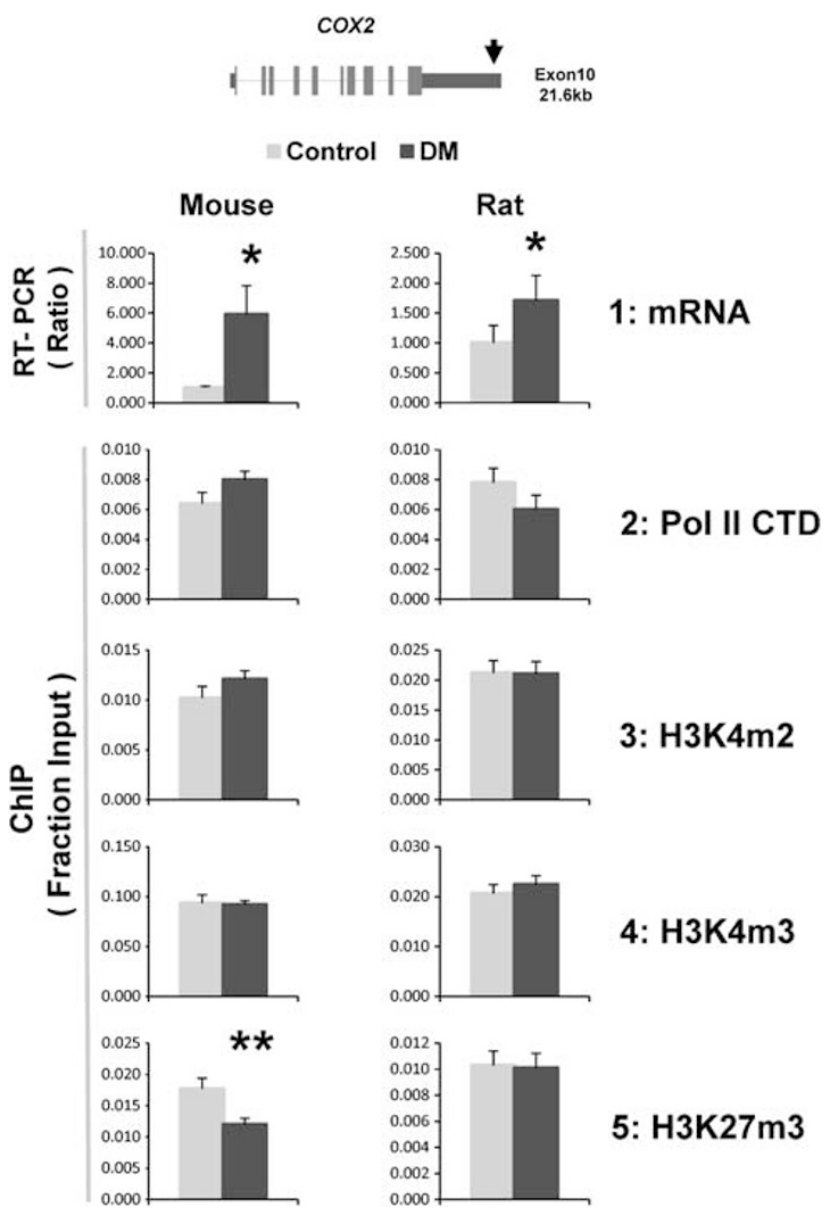

Figure 1 RT-qPCR and Matrix ChIP analysis mouse and rat Cox2 gene in T1D kidneys. All assays were done in age-matched control diabetes in pairwise fashion. RT-qPCR (row 1); total RNA from renal cortex was used in RT reactions with random hexamers. CDNA was used in PCR using gene-specific primers to the last exon of Cox2. The mRNA level of a given gene in each sample was normalized to the level of $\beta$-actin transcript. mRNA data in diabetic animals are expressed as fold of nondiabeticpaired respective normal age-matched controls. ChIP-qPCR (row 2-5); sheared cross-linked kidney chromatin from diabetic animals and their nondiabetic controls were assayed using Pol II CTD (row 2), H3K4m2 (row 3), H3K4m3 (row 4), and H3K27m3 (row 5) antibodies. ChIP DNA was analyzed at the last exon in real-time PCR. Data represent mean \pm s.e.m. (six animals from each group) expressed as fold change of \% of input in diabetic (DM) as compared with nondiabetic control (Control) animals (row $2-5$ ). ${ }^{*} P<0.05$ and ${ }^{* *} P<0.01$. Schematic of the Cox 2 gene is shown above the graphs; exons are shown as rectangles (taller and shorter rectangles represent translated and untranslated regions, respectively); lines represent introns. Arrow shows location of the amplicon.

\section{Monocyte Chemotactic Protein-1 (Mcp-1)}

Similar to Cox2, there were higher levels of mouse Mcp-1 $(\mathrm{mMcp}-1)$ mRNA $(P<0.01)$ in OVE26 compared with FVB animals (Figure 2, row 1). These changes were not associated with significant elevation of Pol II recruitment to the locus (row 2), suggesting that the higher levels of mMCP-1 mRNA expression could be mostly mediated by increased stability of the mMcp-1 transcript in the diabetic mouse kidneys. Consistent with increased mRNA expression (row 1), activating

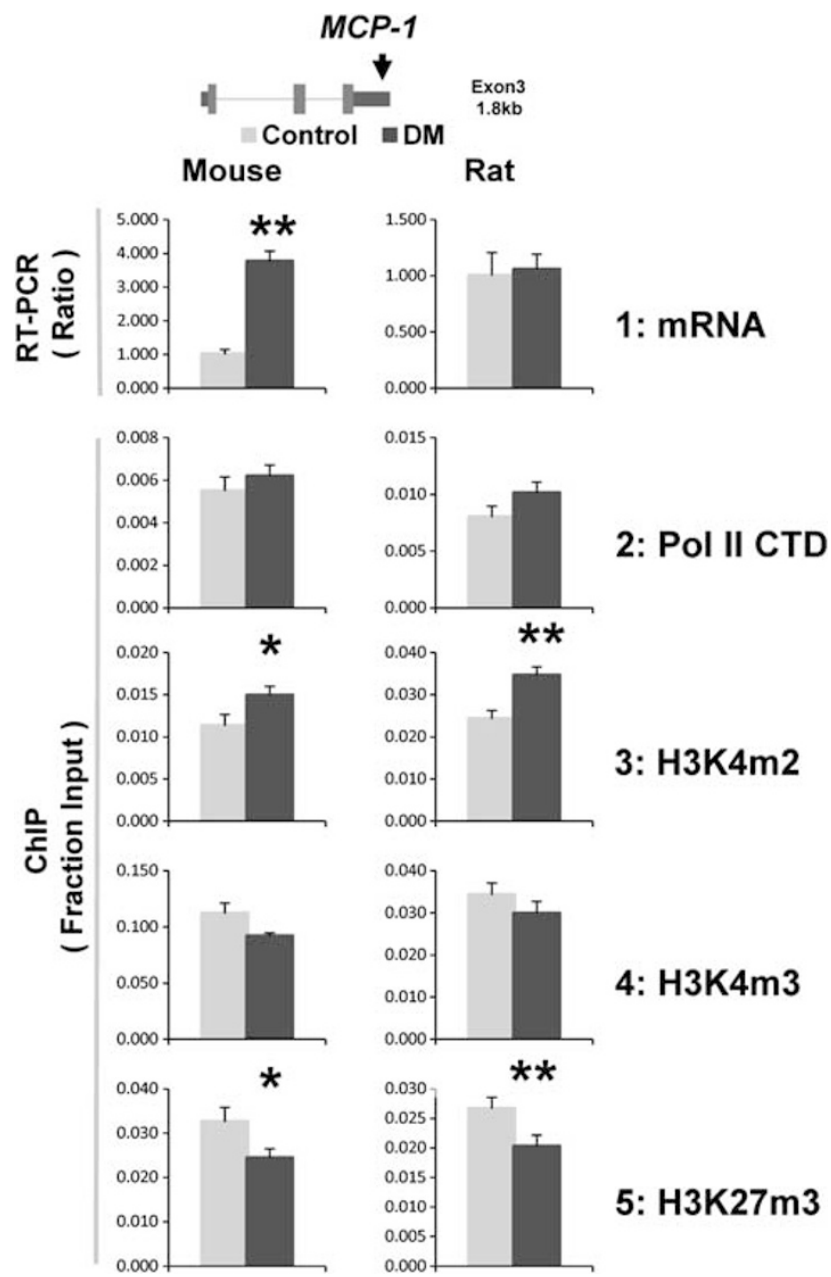

Figure 2 RT-qPCR and Matrix ChIP analysis mouse and rat Mcp-1 gene in T1D kidneys. mRNA data in diabetic animals are expressed as fold of nondiabetic-paired respective normal age-matched controls. ChIP data represent mean \pm s.e.m. (six animals from each group), expressed as fold change of $\%$ of input in diabetic (DM) compared with nondiabetic control (Control) animals (row $2-5$ ). ${ }^{*} P<0.05$ and ${ }^{* *} P<0.01$. Schematic of the Mcp-1 gene is shown above the graphs; exons are shown as rectangles (taller and shorter rectangles represent translated and untranslated regions, respectively); lines represent introns. Arrow shows location of the amplicon.

H3K4m 2 was increased $(P<0.05$, row 3$)$, and, similarly to the $m \operatorname{Cox} 2$ locus, the repressive $\mathrm{H} 3 \mathrm{~K} 27 \mathrm{~m} 3$ mark at the $m M c p-1$ locus was lower in diabetic mice $(P<0.05$, row 5$)$.

In contrast to mouse model, diabetic rats showed no difference in Mcp-1 mRNA expression (rMcp-1) (Figure 2, row 1) as compared with controls. Yet, $\mathrm{H} 3 \mathrm{~K} 4 \mathrm{~m} 2$ and $\mathrm{H} 3 \mathrm{~K} 27 \mathrm{~m} 3$ changes paralleled to those in mice.

\section{S100A4/FSP-1}

S100A4, a member of the S100 family $\mathrm{Ca}^{2+}$-binding proteins, is a multifunctional protein expressed at sites of inflammation, including a spectrum of renal disorders. ${ }^{21}$ S100A4, also known as FSP-1, has been identified as a mesenchymal marker, expressed early in renal fibrosis. ${ }^{21}$ 

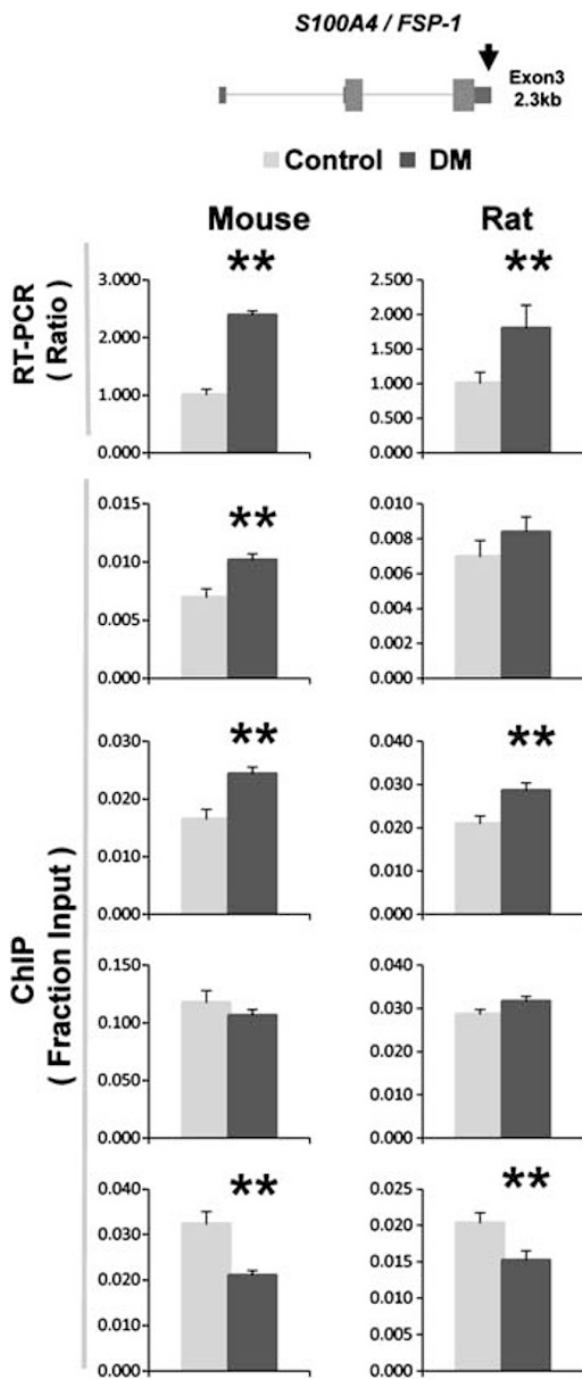

1: mRNA

2: Pol II CTD

3: H3K4m2

4: H3K4m3

\section{5: H3K27m3}

Figure 3 RT-qPCR and Matrix ChIP analysis mouse and rat S100A4/FSP-1 gene in T1D kidneys. mRNA data in diabetic animals are expressed as fold of nondiabetic-paired respective normal age-matched controls. ChIP data represent mean \pm s.e.m. (six animals from each group), expressed as fold change of $\%$ of input in diabetic (DM) compared with nondiabetic control (Control) animals (row $2-5$ ). ${ }^{*} P<0.05$ and ${ }^{* *} P<0.01$. Schematic of the S100A4/FSP-1 gene is shown above the graphs; exons are shown as rectangles (taller and shorter rectangles represent translated and untranslated regions, respectively); lines represent introns. Arrow shows location of the amplicon.

Mouse S100A4/FSP-1 (mS100A4/FSP-1) mRNA expression levels were higher in OVE26 compared with FVB $(P<0.001$, Figure 3 , row 1$)$. These differences were associated with higher Pol II levels at the mS100A4/FSP-1 locus in the OVE26 as compared with controls $(P<0.01)$ (row 2$)$, suggesting that the increase in $m S 100 A 4 / F S P-1$ mRNA levels in the diabetic mice is, at least in part, transcriptionally mediated. Consistent with these findings, the level of an activating mark, $\mathrm{H} 3 \mathrm{~K} 4 \mathrm{~m} 2$ at this locus, was significantly higher $(P<0.01$, row 3), and H3K27m3 levels were lower in the OVE26 compared with FVB mice $(P<0.01$, row 5$)$. These reciprocal changes in $\mathrm{H} 3 \mathrm{~K} 4 \mathrm{~m} 2$ and $\mathrm{H} 3 \mathrm{~K} 27 \mathrm{~m} 3$ levels may contribute to increased transcription of the S100A4/FSP-1 gene in these diabetic mice.

Diabetic rats also demonstrated higher levels of the rat $(\mathrm{rS100A4/FSP-1)}$ mRNA $(P<0.01)$ (Figure 3, row 1). There was some, though insignificant, increase in Pol II levels at the $r S 100 A 4 / F S P-1$ locus between diabetic and nondiabetic rats. $\mathrm{H} 4 \mathrm{~K} 4 \mathrm{~m} 2$ was increased $(P<0.05)$ and $\mathrm{H} 3 \mathrm{~K} 27 \mathrm{~m} 3$ decreased in diabetic rats as compared with controls $(P<0.1)$ (Figure 3, row 5), resembling observations in mice.

\section{Vimentin}

Vim is another mesenchymal marker expressed during tubulointerstitial fibrosis (Figure 4). In OVE26 mice, Vim (mVim) mRNA was higher compared with controls $(P<0.01)$ (Figure 4, row 1$)$. The higher transcript levels were associated with no change in Pol II levels (row 2) and lower H3K27m3 density $(P<0.01$, row 5$)$. In diabetic rats, the Vim mRNA levels were higher than that in controls $(P<0.01$, row $1)$, but these changes were not associated with differences in Pol II or histone marks.

\section{Laminin $\gamma 1$ (Lamc1)}

Tubulointerstitial fibrosis in DN is associated with aberrant accumulation of normal components of extracellular matrix (ECM), including laminin chains. ${ }^{34-35}$ In the ECM, the $\gamma 1$ chain is one of the most widely expressed laminin chains, and its level is increased in DN. ${ }^{23,35}$ mLamc1 mRNA levels were higher in OVE26 compared with FVB controls $(P<0.01)$ (Figure 5, row 1). These changes were matched by an increase in $\mathrm{H} 3 \mathrm{~K} 4 \mathrm{~m} 2$ levels (row 3 ) and by a decrease in silencing H3K27m3 levels $(P<0.01$, (row 5). No measurable difference between control and diabetic rats in mRNA, Pol II, and histone marks were seen for Lamcl gene.

\section{Cyclophiline A}

Cyclophiline A (CypA) is an abundant, ubiquitously expressed housekeeping protein. ${ }^{36}$ mRNA and Pol II levels tended to be higher in the diabetic OVE26 mice, but these differences were not statistically significant. (Figure 6, row 1$2)$. $\mathrm{H} 3 \mathrm{~K} 4 \mathrm{~m} 2$ density was higher $(P<0.05$, row 3$)$ and H3K27m3 density was lower $(P<0.05)$ (row 5) at CypA gene in the diabetic mice. No measurable differences between control and diabetic rats in mRNA, Pol II, and histone marks were seen for CypA gene.

\section{Expression of Histone-modifying Enzymes}

Diabetes-induced epigenetic changes at a given locus could reflect altered rates of recruitment of chromatin-modifying enzymes or be caused by changes in cellular levels and/or activity of these proteins. In the above experiments, lower levels of the repressive $\mathrm{H} 3 \mathrm{~K} 27 \mathrm{~m} 3$ mark appeared to be the most consistent epigenetic change observed in diabetic kidneys of these models. This phenomenon could be due to diabetes-induced alterations in the levels of the histone 


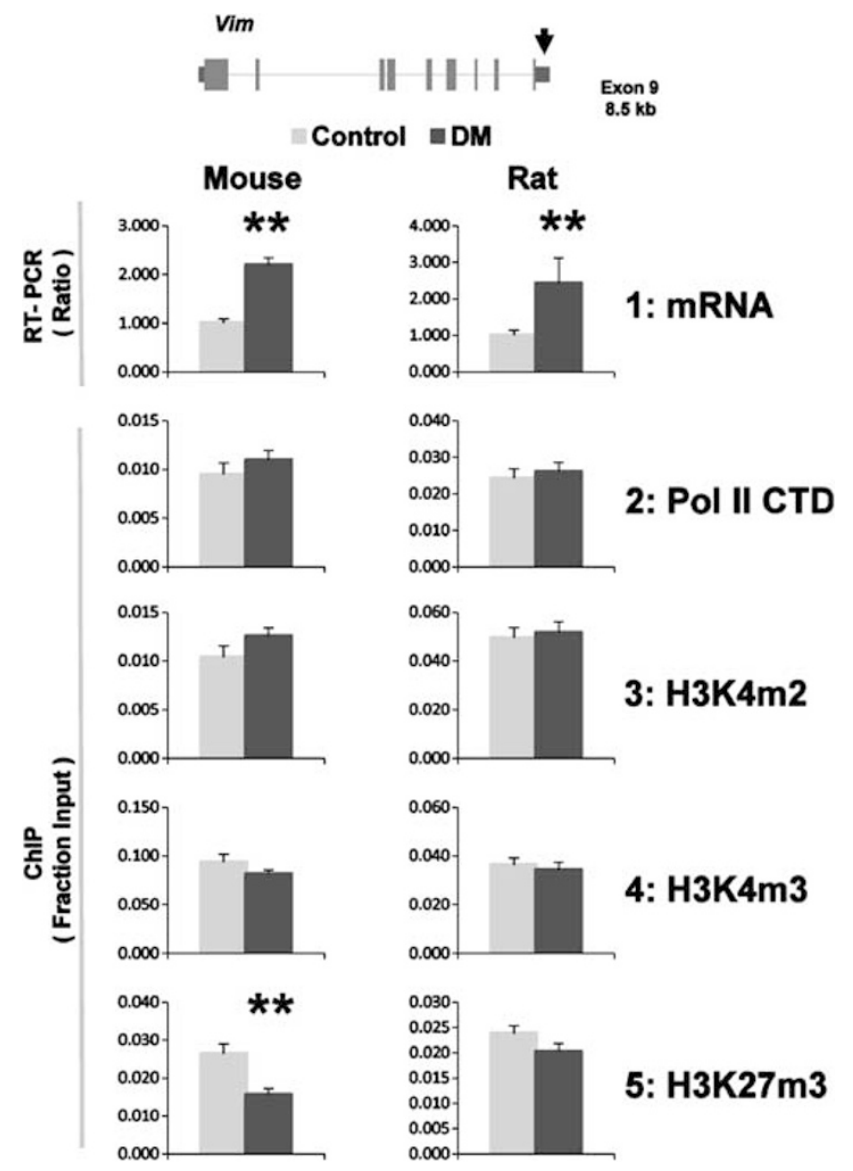

Figure 4 RT-qPCR and Matrix ChIP analysis mouse and rat Vim gene in T1D kidneys. mRNA data in diabetic animals are expressed as fold of nondiabetic-paired respective normal age-matched controls. ChIP data represent mean \pm s.e.m. (six animals from each group), expressed as fold change of $\%$ of input in diabetic (DM) compared with nondiabetic control (Control) animals (row $2-5$ ). ${ }^{*} P<0.05$ and ${ }^{* *} P<0.01$. Schematic of the Vim gene is shown above the graphs; exons are shown as rectangles (taller and shorter rectangles represent translated and untranslated regions, respectively); lines represent introns. Arrow shows location of the amplicon.

demethylases (KDM6) ${ }^{37-38}$ and/or methyltransferase (Ezh2) enzymes that modify this histone $\mathrm{H} 3$ lysine residue. ${ }^{39}$ This possibility was examined using immunoblot analysis. As shown in Figure 7a, examination of whole-mouse kidney homogenates revealed increased protein abundance of KDM6A in diabetic OVE26 $(P<0.05$, left panel). However, little or no differences were observed in rat kidney samples (Figure 7a, right panel), even though rats also demonstrated diabetes-induced decreases in $\mathrm{H} 3 \mathrm{~K} 27 \mathrm{~m} 3$ at some genes. The Ezh2 protein expression was not different between the OVE26 and control mice (Figure 7b, left panel). In contrast, kidneys of diabetic rats demonstrated higher Ezh2 levels $(P<0.05$, right panel).

\section{DISCUSSION}

We used RT-PCR, multiplex Matrix ChIP, and western blot analyses of histone-modifying enzymes to present the first of
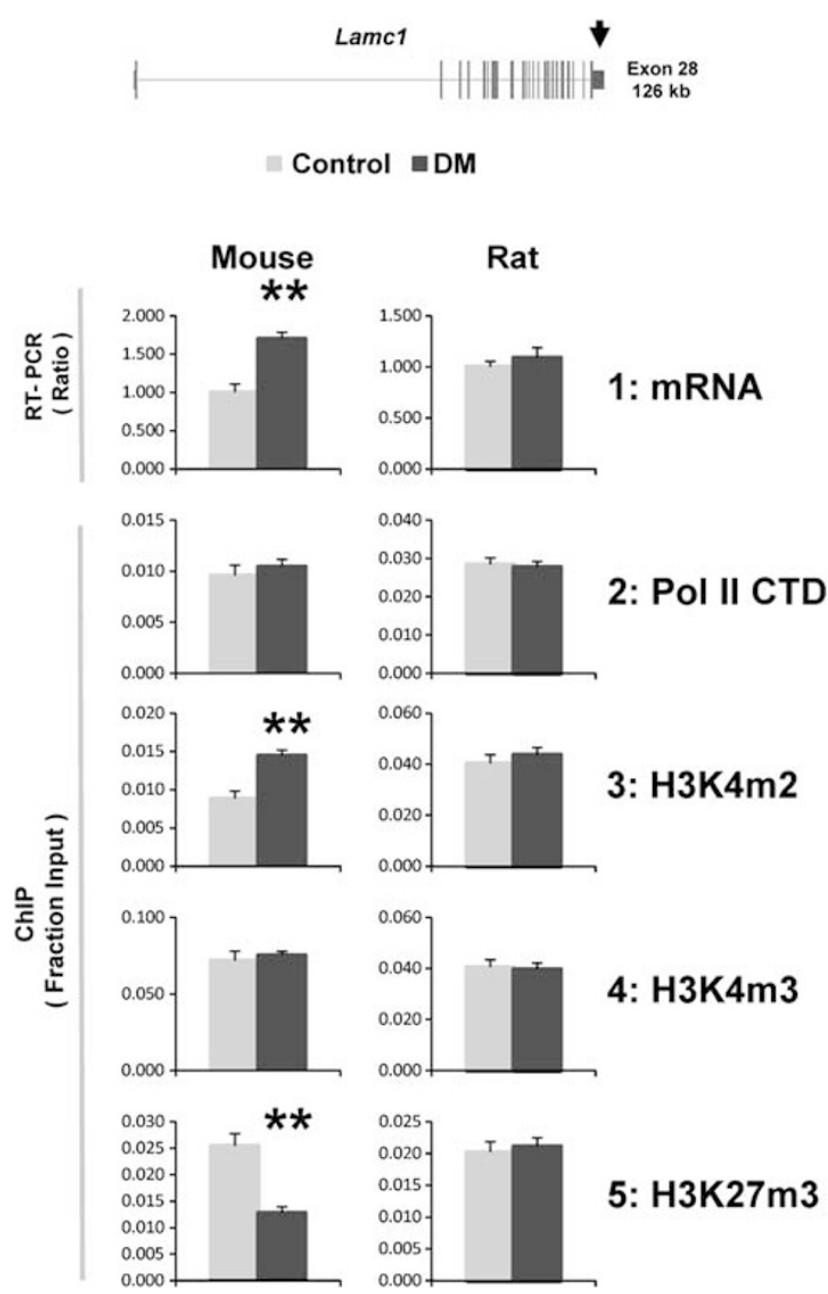

4: H3K4m3

Figure 5 RT-qPCR and Matrix ChIP analysis mouse and rat Lamc1 gene in T1D kidneys. mRNA data in diabetic animals are expressed as fold of nondiabetic-paired respective normal age-matched controls. ChIP data represent mean \pm s.e.m. (six animals from each group), expressed as fold change of $\%$ of input in diabetic (DM) compared with nondiabetic control (Control) animals (row $2-5$ ). ${ }^{*} P<0.05$ and ${ }^{* *} P<0.01$. Schematic of the Lamc1 gene is shown above the graphs; exons are shown as rectangles (taller and shorter rectangles represent translated and untranslated regions, respectively); lines represent introns. Arrow shows location of the amplicon.

its kind parallel determination of renal gene expression and epigenetic analyses in kidneys collected from two different rodent models of diabetes at DN-relevant loci. The most consistent epigenetic finding at the diabetes-induced renal genes in both mice and rats was a decrease in repressive H3K27m3 mark levels. Although this specific epigenetic change has not previously been reported in diabetes, it is consistent with observations that the diabetic milieu renders chromatin structure more accessible to transcriptional machinery. $1,7,12,28,40$ We also observed increased levels of the activating epigenetic mark $\mathrm{H} 3 \mathrm{~K} 4 \mathrm{~m} 2$ in both the diabetic mice and rats. Differences in $\mathrm{H} 3 \mathrm{~K} 4 \mathrm{~m} 2$ at pro-inflammatory genes between normal and diabetic animals have been previously reported in CVS studies. ${ }^{3}$ 

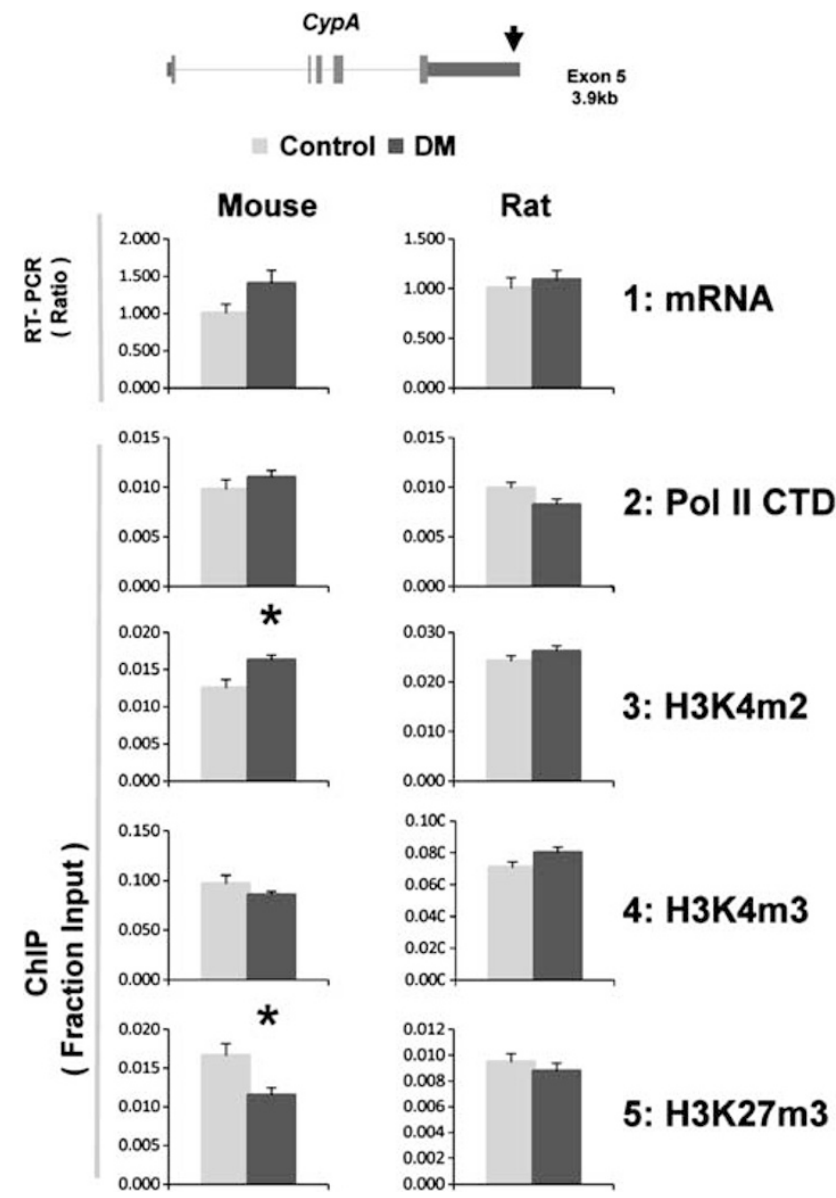

Figure 6 RT-qPCR and Matrix ChIP analysis mouse and rat CypA gene in T1D kidneys. mRNA data in diabetic animals are expressed as fold of nondiabetic-paired respective normal age-matched controls. ChIP data represent mean \pm s.e.m. (six animals from each group), expressed as fold change of $\%$ of input in diabetic (DM) compared with nondiabetic control (Control) animals (row $2-5$ ). ${ }^{*} P<0.05$ and ${ }^{* *} P<0.01$. Schematic of the CypA gene is shown above the graphs; exons are shown as rectangles (taller and shorter rectangles represent translated and untranslated regions, respectively); lines represent introns. Arrow shows location of the amplicon.

Chromatin modifications reflect a balance of activities between so-called 'writer' and 'eraser' enzymes (eg, methylases and demethylases ${ }^{39}$ ). Changes in modification of a given histone residue could reflect changes in level of their expression of corresponding enzymes, their catalytic activity, and/or their binding to genes (or all of these determinants). There are examples where the diabetic milieu has been shown to alter whole nuclear patterns of histone modifications, reflecting changes in the expression of histone-modifying enzymes. ${ }^{9,41}$ By immunoblot analysis, we found that the protein levels of one of the two known H3K27m2/3 demethylases, KDM6A, ${ }^{39}$ was higher in kidneys of the diabetic OVE26 mice compared with controls (Figure $7 \mathrm{a}$, left panel). In contrast, protein levels of the key H3K27 methyltransferase Ezh2 ${ }^{42}$ were not different between normal and diabetic mice (Figure $7 \mathrm{~b}$, left panel). It seems plausible that elevated KDM6A could be responsible for erasing the repressive $\mathrm{H} 3 \mathrm{~K} 27 \mathrm{~m} 3$ modification at some genes, leading to a more open chromatin structure and, therefore, facilitating Pol II transcriptional output in this mouse model. Thus, in the OVE26 mice, increased expression of KDM6A could be one of the primary changes triggered by the diabetic milieu that initiate the chain of events leading to the abnormally high levels of some mRNAs seen in this mouse DN model.

Although there are substantial differences between the two models of T1D used in this work, we reasoned that a parallel study would be helpful to provide epigenetic information with general applicability to the diabetic kidney. Indeed, we found some similarities in the mouse and rat models exemplified by S100A4/FSP-1 mRNA increases that were associated with increased Pol II density and decreased $\mathrm{H} 3 \mathrm{~K} 27 \mathrm{~m} 3$ levels (Figure 3). Nonetheless, the diabetic mice displayed greater changes in mRNA expression and corresponding chromatin modifications compared with rats. Moreover, there were clear differences in alterations in H3K27-modifying enzymes between the two species (Figure 7). These differences between the two models may reflect species specificity of responses to the diabetic milieu and secondary compensatory responses in the kidney. For example, the OVE26 mice display a more aggressive course of diabetes than rats, with BG levels approaching $700 \mathrm{mg} / \mathrm{dl}$. This factor alone can have a role, as the expression of histone-modifying enzymes can be altered by high glucose. ${ }^{43,44}$ In contrast, the rat model displays fluctuating BG levels because of suboptimal insulin treatment and, at least, temporary residual insulin secretion. From the metabolic point of view, the rat model is more clinically relevant, but may not be sufficiently aggressive to induce consistent robust chromatin changes during several weeks of hyperglycemia. The fact that, unlike in mice, KDM6A levels were not altered in diabetic rats may reflect less-severe hyperglycemia in the STZ model.

In addition, the role of insulin in rats, though administered in suboptimal doses, together with residual insulinemia present for several weeks after STZ injection, cannot also be excluded. Insulin has been shown to induce chromatin changes in typical insulin-sensitive cells, such as adipocytes. ${ }^{45}$ Moreover, our own studies identified direct epigenetic roles for insulin receptor in liver cells, ${ }^{28}$ which could be also operating in the kidney. The interaction of components of diabetic milieu with insulin in altering histone modifications in diabetic cells is one of the directions for further research in this field. Yet, available experience in vascular cells obtained from $\mathrm{db} / \mathrm{db}$ mice that display both high glucose and insulin levels suggests that in models of diabetes, hyperglycemia is the major driving force of epigenetic changes, at least, along the pro-inflammatory genes. ${ }^{3,46}$ Clearly, more studies are needed to dissect out the contribution of the different components of diabetic milieu to epigenetic changes. In this regard, expanding epigenetic comparisons to other DN models and human disease promises to be helpful. 

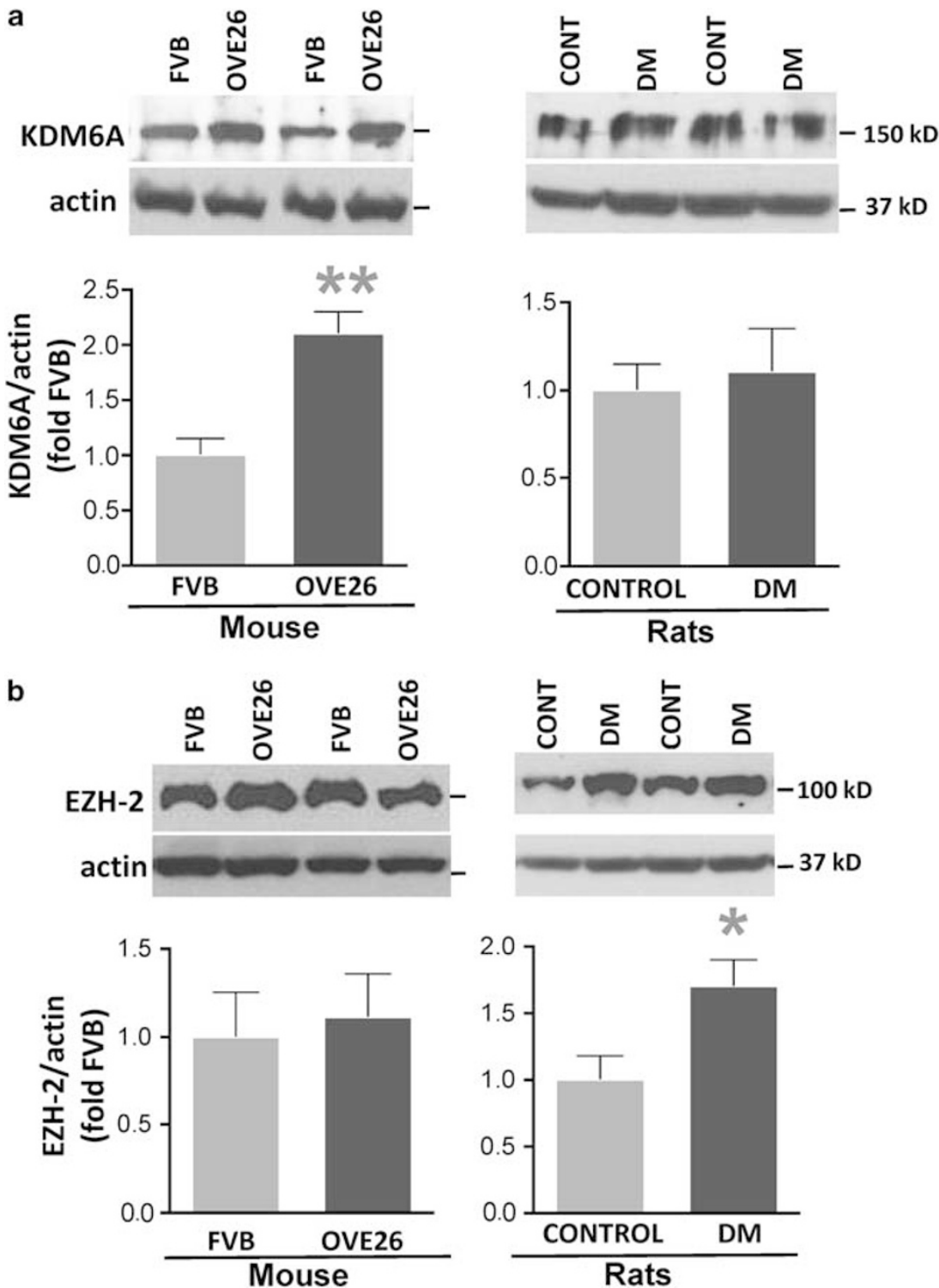

Figure 7 Western blot analysis of KDM6A demethylase and Ezh2 methyltransferase in mouse and rat T1D kidney homogenates. (a) Representative blots and densitometry $(n=6)$ of renal KDM6A protein abundance in OVE26 and control FVB mice and STZ diabetic rats. (b) Representative blots and densitometry $(n=6)$ of renal Ezh2 protein abundance in OVE26 and control FVB mice and STZ diabetic rats. $\left({ }^{*} P<0.05\right.$ vs control rats; ${ }^{* *} P<0.01$ vs FVB mice).

Measurements of both Pol II at a gene and the cognate transcript levels in the same sample provide a way to assess contribution of transcriptional and posttranscriptional processes to mRNA expression. ${ }^{47}$ Our present data support a substantial role of posttranscriptional processes in diabetesinduced mRNA changes, because no changes in Pol II levels in both mice and rats were seen at some diabetes-induced genes (Figures 1-6). Shanmugam et al. ${ }^{48}$ demonstrated that diabetic stimuli can stabilize mRNAs encoding proinflammatory mediators, including COX2. Thus, it is likely that in our models, diabetes increased stability of some of the renal transcripts examined in the current study. Furthermore, message degradation is regulated by RNA-binding proteins and microRNAs. ${ }^{48}$ Diabetes can downregulate some
microRNAs, resulting in the increased expression of target mRNAs, ${ }^{49}$ a scenario that may explain higher levels of mRNA in the diabetic animals without corresponding increases in bound Pol II.

The current study utilized kidney cortical samples. The kidney is a heterogeneous organ, and we expect that some of the chromatin changes during disease progression might be cell-type specific. About $90 \%$ of cortex is formed by renal tubular cells-therefore, our present observations are most relevant to these cell types. In vitro studies could provide celltype-specific information, but do not faithfully recapitulate the in vivo duration and complexity of the diabetic milieu. Approaches to gain cell-specific epigenetic information are possible using microcapture or microdissection of individual 
cells, but further technological advances are needed in ChIP assays to examine small kidney cell populations.

Until now, the majority of epigenetic studies, including those relevant to diabetes, ${ }^{5,44,50}$ have been done in cell cultures. Compared with in vitro studies, ${ }^{12}$ the extent of diabetes-induced epigenetic changes observed in the current study in vivo is smaller. One possible reason for the differences in the magnitude of changes could be that, as mentioned above, only certain cell types do respond to diabetic milieu that represent a small fraction of renal cortex. In addition, unlike cell cultures that are generally maintained in stable environment (eg, normal vs high glucose), in vivo conditions are fluctuating. The magnitude of in vivo epigenetic changes observed here are also smaller than the alterations we have previously reported in association with acute kidney injury following renal ischemia. ${ }^{14,51}$ Although the impact of changes described here on the progression of DN remains to be defined, epigenetic alterations resulting in small increases in rates of Pol II elongation induced by diabetes could cause accumulation of cognate mRNAs (eg, Cox2, Mcp-1, S100A4/FSP-1, and Vim). Higher and sustained protein levels encoded by these transcripts could have significant biological implications in DN.

Recent technological advances, such as ChIP-Seq, are providing unprecedented means to profile epigenetic marks genome-wide. ${ }^{33,52}$ As the cost of such studies becomes more affordable, and user friendly tools to analyze the large amount of data become available, whole-genome screens will provide new avenues to explore epigenetics of $\mathrm{DN}$.

In summary, we show that altered mRNA expression of some DN-related genes is associated with changes in Pol II recruitment and chromatin marks at these selected loci. Decreased levels of repressive $(\mathrm{H} 3 \mathrm{~K} 27 \mathrm{~m} 3)$, and increased levels of activating $(\mathrm{H} 3 \mathrm{~K} 4 \mathrm{~m} 2)$, epigenetic marks were the most consistent finding in both mouse and rat diabetic models. The observed decrease in mouse $\mathrm{H} 3 \mathrm{~K} 27 \mathrm{~m} 3$ was consistent with higher cellular levels of the cognate demethylase KDM6A. Finally, our results indicate that both altered transcription and posttranscriptional mechanisms could mediate increased expression of renal genes in experimental models of diabetes.

\section{ACKNOWLEDGEMENTS}

This work was supported by JDRF grant 42-2009-779, NIH grants R37 DK45978 and R01 DK083310, and an anonymous private donation to UW Medicine Research.

\section{DISCLOSURE/CONFLICT OF INTEREST}

The authors declare no conflict of interest.

1. Brasacchio D, Okabe J, Tikellis C, et al. Hyperglycemia induces a dynamic cooperativity of histone methylase and demethylase enzymes associated with gene-activating epigenetic ks that co-exist on the lysine tail. Diabetes 2009;58:1229-1236.

2. Villeneuve $L M$, Reddy $M A$, Lanting $L L$, et al. Epigenetic histone $H 3$ lysine 9 methylation in metabolic memory and inflammatory phenotype of vascular smooth muscle cells in diabetes. Proc Nat Acad Sci USA 2008;105:9047-9052.

3. Reddy MA, Villeneuve LM, Wang $M$, et al. Role of the lysine-specific demethylase 1 in the proinflammatory phenotype of vascular smooth muscle cells of diabetic mice. Circ Res 2008;103:615-623.

4. Liu Y, Dentin R, Chen D, et al. A fasting inducible switch modulates gluconeogenesis via activator/coactivator exchange. Nature 2008;456: 269-273.

5. El-Osta A, Brasacchio D, Yao D, et al. Transient high glucose causes persistent epigenetic changes and altered gene expression during subsequent normoglycemia. J Exp Med 2008;205:2409-2417.

6. Miao F, Wu X, Zhang L, et al. Histone methylation patterns are celltype specific in human monocytes and lymphocytes and well maintained at core genes. J Immunol 2008;180:2264-2269.

7. Miao $F$, Gonzalo IG, Lanting $L$, et al. In vivo chromatin remodeling events leading to inflammatory gene transcription under diabetic conditions. J Biol Chem 2004;279:18091-18097.

8. Lee HB, Noh H, Seo JY, et al. Histone deacetylase inhibitors: a el class of therapeutic agents in diabetic nephropathy. Kidney Int Suppl 2007; 106:S61-S66.

9. Tikoo K, Meena RL, Kabra DG, et al. Change in post-translational modifications of histone $\mathrm{H} 3$, heat-shock protein-27 and MAP kinase p38 expression by curcumin in streptozotocin-induced type I diabetic nephropathy. Br J Pharmacol 2008;153:1225-1231.

10. Miao F, Natara R. Mapping global histone methylation patterns in the coding regions of human genes. Mol Cell Biol 2005;25:4650-4661.

11. Miao F, Wu X, Zhang L, et al. Genome-wide analysis of histone lysine methylation variations caused by diabetic conditions in human monocytes. J Biol Chem 2007;282:13854-13863.

12. Sun $G$, Reddy $M A$, Yuan $H$, et al. Epigenetic histone methylation modulates fibrotic gene expression. J Am Soc Nephrol 2010;21: 2069-2080.

13. Zheng S, Noonan WT, Metreveli NS, et al. Development of late-stage diabetic nephropathy in OVE26 diabetic mice. Diabetes 2004;53: 3248-3257.

14. Naito M, Zager RA, Bomsztyk K. BRG1 increases transcription of proinflammatory genes in renal ischemia. J Am Soc Nephrol 2009; 20:1787-1796.

15. Nelson JD, Flanagin $S$, Kawata $Y$, et al. Transcription of laminin $\gamma 1$ chain gene in rat mesangial cells: constitutive and inducible RNA polymerase II recruitment and chromatin states.. Am J Physiol Renal Physiol 2008;294:F525-F533.

16. Komers R, Oyama $T$, Beard DR, et al. Rho kinase inhibition protects kidneys from diabetic nephropathy without reducing blood pressure. Kidney Int 2011;79:432-442.

17. Komers R, Lindsley JN, Oyama TT, et al. Cyclo-oxygenase-2 inhibition attenuates the progression of nephropathy in uninephrectomized diabetic rats. Clin Exp Pharmacol Physiol 2007;34:36-41.

18. Komers R, Lindsley JN, Oyama TT, et al. Immunohistochemical and functional correlations of renal cyclooxygenase- 2 in experimental diabetes. J Clin Invest 2001;107:889-898.

19. Wang J-L, Cheng H-F, Shappell S, et al. Cyclooxygenase-2 (COX-2) inhibition reases proteinuria in a hypertensive diabetic model. J Amer Soc Nephrol 1999;10:A3505.

20. Tesch GH. MCP-1/CCL2: a new diagnostic ker and therapeutic target for progressive renal injury in diabetic nephropathy. Am J Physiol Renal Physiol 2008;294:F697-F701.

21. Rossini $M$, Cheunsuchon $B$, Donnert $E$, et al. Immunolocalization of fibroblast growth factor-1 (FGF-1), its receptor (FGFR-1), and fibroblast-specific protein-1 (FSP-1) in inflammatory renal disease. Kidney Int 2005;68:2621-2628.

22. Coimbra TM, ssen U, Grone HJ, et al. Early events leading to renal injury in obese Zucker (fatty) rats with type II diabetes. Kidney Int 2000;57:167-182.

23. Setty $\mathrm{S}$, Michael AA, Fish AJ, et al. Differential expression of laminin isoforms in diabetic nephropathy and other renal diseases. Mod Pathol 2012;25:859-868.

24. Eid AA, Ford BM, Block $K$, et al. AMPK negatively regulates NOX4dependent activation of p53 and epithelial cell apoptosis in diabetes. J Biol Chem 2010;285:37503-37512.

25. Xu J, Huang $\mathrm{Y}, \mathrm{Li} F$, et al. FVB mouse genotype confers susceptibility to OVE26 diabetic albuminuria. Am J Physiol Renal Physiol 2010;299: F487-F494. 
26. Nelson JD, Denisenko $O$, Sova $P$, et al. Fast chromatin immunoprecipitation assay. Nucleic Acids Res. 2006;34:e2.

27. Flanagin S, Nelson JD, Castner DG, et al. Microplate-based chromatin immunoprecipitation method, Matrix ChIP: a platform to study signaling of complex genomic events. Nucleic Acids Res 2008;36:e17.

28. Nelson JD, Leboeuf RC, Bomsztyk K. Direct recruitment of insulin receptor and ERK signaling cascade to insulin-inducible gene loci. Diabetes 2011;60:127-137.

29. Komers R, Schutzer WE, Reed JF, et al. Altered endothelial nitric oxide synthase targeting and conformation and caveolin-1 expression in the diabetic kidney. Diabetes 2006;55:1651-1659.

30. Cheng HF, Wang CJ, Moeckel GW, et al. Cyclooxygenase-2 inhibitor blocks expression of mediators of renal injury in a model of diabetes and hypertension. Kidney Int 2002;62:929-939.

31. Giunti S, Tesch GH, Pinach S, et al. Monocyte chemoattractant protein1 has prosclerotic effects both in a mouse model of experimental diabetes and in vitro in human mesangial cells. Diabetologia 2008; 51:198-207.

32. Hargreaves DC, Horng T, Medzhitov R. Control of inducible gene expression by signal-dependent transcriptional elongation. Cell 2009; 138:129-145.

33. Barski A, Cuddapah S, Cui K, et al. High-resolution profiling of histone methylations in the human genome. Cell 2007;129:823-837.

34. Phillips SL, DeRubertis FR, Craven PA. Regulation of the laminin C1 promoter in cultured mesangial cells. Diabetes 1999;48:2083-2089.

35. Yang CW, Hattori $M$, Vlassara $H$, et al. Overexpression of transforming growth factor-beta $1 \mathrm{mRNA}$ is associated with up-regulation of glomerular tenascin and laminin gene expression in nonobese diabetic mice. J Am Soc Nephrol 1995;5:1610-1617.

36. Schmid $H$, Cohen $C D$, Henger $A$, et al. Validation of endogenous controls for gene expression analysis in microdissected human renal biopsies. Kidney Int 2003;64:356-360.

37. Swigut T, Wysocka J. H3K27 demethylases, at long last. Cell 2007; 131:29-32.

38. De Santa F, Totaro MG, Prosperini E, et al. The histone H3 lysine-27 demethylase Jmjd3 links inflammation to inhibition of polycombmediated gene silencing. Cell 2007;130:1083-1094.

39. Allis $C D$, Berger $S L$, Cote J, et al. New nomenclature for chromatinmodifying enzymes. Cell 2007;131:633-636.

40. Villeneuve LM, Reddy MA, Natarajan R. Epigenetics: iphering its role in diabetes and its chronic complications. Clin Exp Pharmacol Physiol 2011:38:401-409.
41. Sayyed SG, Gaikwad AB, Lichtnekert J, et al. Progressive glomerulosclerosis in type 2 diabetes is associated with renal histone $\mathrm{H} 3 \mathrm{~K} 9$ and $\mathrm{H} 3 \mathrm{~K} 23$ acetylation, H3K4 dimethylation and phosphorylation at serine 10. Nephrol Dial Transplant 2010;25:1811-1817.

42. Cao $\mathrm{R}$, Wang $\mathrm{L}$, Wang $\mathrm{H}$, et al. Role of histone $\mathrm{H} 3$ lysine 27 methylation in Polycomb-group silencing. Science 2002;298:1039-1043.

43. Naito M, Shenoy A, Aoyama I, et al. High ambient glucose ments angiotensin ii-induced proinflammatory gene mRNA expression in human mesangial cells: effects of valsartan and simvastatin. Am J Nephrol 2009;30:99-111.

44. Okabe J, Orlowski C, Balcerczyk A, et al. Distinguishing hyperglycemic changes by Set7 in vascular endothelial cells. Circ Res 2012;110: 1067-1076.

45. Gupta J, Tikoo K. Involvement of insulin-induced reversible chromatin remodeling in altering the expression of oxidative stress-responsive genes under hyperglycemia in 3T3-L1 preadipocytes. Gene 2012;504: 181-191.

46. Villeneuve LM, Kato M, Reddy MA, et al. Enhanced levels of microRNA$125 \mathrm{~b}$ in vascular smooth muscle cells of diabetic $\mathrm{db} / \mathrm{db}$ mice lead to increased inflammatory gene expression by targeting the histone methyltransferase Suv39h1. Diabetes 2010;59:2904-2915.

47. Nelson J, Denisenko O, Bomsztyk K. Profiling RNA polymerase II using the fast chromatin immunoprecipitation method. Methods Mol Biol 2011;703:219-234.

48. Shanmugam N, Reddy MA, Natarajan R. Distinct roles of heterogeneous nuclear ribonuclear protein $\mathrm{K}$ and microRNA-16 in cyclooxygenase-2 RNA stability induced by $\mathrm{S100b}$, a ligand of the receptor for advanced glycation end products. J Biol Chem 2008;283:36221-36233.

49. Wang B, Komers R, Carew R, et al. Suppression of microRNA-29 expression by TGF-beta1 promotes collagen expression and renal fibrosis. J Am Soc Nephrol 2012;23:252-265.

50. Li $\mathrm{Y}$, Reddy MA, Miao F, et al. Role of the histone H3 lysine 4 methyltransferase, SET7/9, in the regulation of NF-kappaB-dependent inflammatory genes. Relevance to diabetes and inflammation. J Biol Chem 2008;283:26771-26781.

51. Naito M, Bomsztyk K, Zager RA. Renal ischemia-induced cholesterol loading: transcription factor recruitment and chromatin remodeling along the HMG CoA reductase gene. Am J Pathol 2009; 174:54-62.

52. Euskirchen $\mathrm{GM}$, Rozowsky JS, Wei $\mathrm{CL}$, et al. Mapping of transcription factor binding regions in mammalian cells by ChIP: comparison of arrayand sequencing-based technologies. Genome Res 2007;17:898-909. 